\title{
BUBBLE DYNAMICS AND HEAT TRANSFER ON BIPHILIC SURFACES
}

\author{
P. Pontes ${ }^{1}$, R. Cautela ${ }^{1}$, E. Teodori ${ }^{2}$, A. S. Moita ${ }^{*}$, A. Georgoulas ${ }^{3}$, António L. N. Moreira ${ }^{1}$ \\ ${ }^{1} \mathrm{IN}+$ Center for Innovation, Technology and Policy Research, Instituto Superior Técnico, Universidade de Lisboa, \\ Av. Rovisco Pais, 1049-001 Lisboa, Portugal \\ ${ }^{2}$ ASML Holding N.V, De Run 6501, 5504 DR, Veldhoven, The Netherlands \\ ${ }^{3}$ Advanced Engineering Centre, School of Computing, Engineering and Mathematics, Cockcroft Building, \\ Lewes Road, University of Brighton, Brighton BN2 4GJ, UK
}

\section{INTRODUCTION}

Pool boiling has been proven as a very effective process for heat transfer in cooling applications. Surface wettability plays a vital role in pool boiling heat transfer [1]. Surfaces with customized wetting patterns (hydrophilic surfaces with hydrophobic regions), when properly optimized geometrically, have shown a high potential of enhancing heat transfer [2], by promoting nucleation at lower superheat values and allowing a significant increase in the critical heat flux [3]. However, the scarce number of experimental studies performed so far shows a clear limitation: while authors describe nucleation and bubbles dynamics (and eventually thermal) behavior, with significant detail, very little is known regarding the internal flow inside the bubbles, which nevertheless may strongly affect both dynamic and thermal processes. Hence, a deeper knowledge on the dynamic behavior of both the vapor inside the bubble and the surrounding liquid could provide a deeper insight on the forces acting on the bubble. Such detailed description also allows a clearer analysis of the relation between the geometry and varying wettability patterns of the surface and its performance in terms of an effective heat transfer enhancement. In previous works, a two-phase, CFD, enhanced numerical model for nucleate boiling that uses an enhanced Volume of Fluid (VOF) based method [4] has been developed. In this work, this model will be tested in pool boiling applications for biphilic surfaces and validated based on experimental values.

\section{EXPERIMENTAL METHDOLOGY}

The utilised validation data for the proposed numerical model are based on results obtained combining highspeed imaging and timely resolved thermography, following our previous work [5]. The recorded videos are processed using in-house MATLAB routines. Experimental values are averaged from four events.

The experimental setup consisted of a water filled tank with lateral glass windows for optical access. The water inside the tank is kept at saturation temperature at atmospheric pressure with the help of resistance heaters. Test conditions are controlled by K-type thermocouples and a pressure transducer (OMEGA DYNE INC).

The surface is a $20 \mu \mathrm{m}$ thick stainless-steel thin foil (AISI304) that is heated by Joule effect, directly fed by DC current, using a HP6274B power supply. For this part of the study, the simple biphilic pattern consisting of a superhydrophobic circular region in the middle of a hydrophilic stainless-steel surface is utilised. A mask with a single circular region was placed on top of the metal sheet and then coated with a superhydrophobic cover for this purpose.

\section{NUMERICAL METHODOLOGY}

For this study, a previously developed, enhanced VOF-based numerical simulation framework, implemented in the general context of OpenFOAM CFD Toolbox, was utilised. To validate the numerical model the conditions presented in Table 1 were fixed both in the numerical simulation as well as in the laboratory experiments. 
Table 1. Parameters of the experiments and numerical simulation

\begin{tabular}{lc}
\hline \multicolumn{1}{c}{ Parameters } & Values \\
\hline Applied heat flux & $1.39 \mathrm{E} 3\left[\mathrm{~W} / \mathrm{m}^{2}\right]$ \\
Hydrophilic region wettability characterization: & \\
Advancing Contact Angle & $85.54\left[^{\circ}\right]$ \\
Receding Contact Angle & $34.37\left[^{\circ}\right]$ \\
Super Hydrophobic region wettability characterization: & \\
Advancing Contact Angle & $160.88\left[^{\circ}\right]$ \\
Receding Contact Angle & $158.98\left[\left[^{\circ}\right]\right.$ \\
Region diameter & $1.5[\mathrm{~mm}]$ \\
\hline
\end{tabular}

In the VOF method, a volume fraction field $\alpha$ identifies the volume of liquid within a cell. The volume of the gaseous phase is therefore given as $(1-\alpha)$. The value of $\alpha$ is 1 inside the pure liquid cells, 0 in the pure gas cells and between 0 and 1 in the cells containing the interface area. This procedure allows using a single set of continuity and momentum equations for the entire flow domain. A more detailed description of the utilised numerical simulation framework can be found in [4]. For the purposes of the present numerical investigation 2D axisymmetric numerical simulations were performed. For this purpose a wedge computational domain representing a $5^{\circ}$ section of the actual 3D phenomenon was utilised. A non-uniform, structured computational mesh was constructed consisting of a total number of 59,848 cells. The mash and the vertical and horizontal dimensions of the computational domain were selected after appropriate mesh independency and domain independency studies.

\section{RESULTS}

Figure 1 depicts a comparison between the experimental results and the model predictions. Figure 1 (a), shows the visual pairing of the experimental and the numerical images at several stages of the considered bubble growth. This first analysis shows a very good agreement between the experimental and the numerical data and a very promising numerical reproduction of the bubble shape, even at later stages of bubble detachment $(\mathrm{t}=2.020 \mathrm{~s}$ and $\mathrm{t}=2.024 \mathrm{~s})$ where necking is more pronounced. Figure $1(\mathrm{~b})$ shows the comparison between the temporal variation of the equivalent bubble diameter. This diameter is calculated using the bubble volume and assuming a spherical approximation. In this case, there is a very good agreement between the experimental data and the numerical results. At later stages of bubble detachment, the results for both the volume and maximum diameter are considerably closer than at earlier stages. This may be due to the fact that, at instant 0 , the experiments start with a vapor layer that covers all the superhydrophobic region. This is due to the fact that several bubble cycles have preceded the considered bubble growth and detachment. The numerical model on the other hand starts with no vapor layer and only one bubble growth and detachment cycle is considered. Regarding the bubble detachment time, the fact that there is no vapor layer at the initial instant for the numerical simulation was considered in the graph by displacing the experimental results to the time when the numerical diameter reached the layer diameter. Hence, the final detachment time observed in both experiments and numerical predictions is the same, showing less than 0.1 seconds of difference.

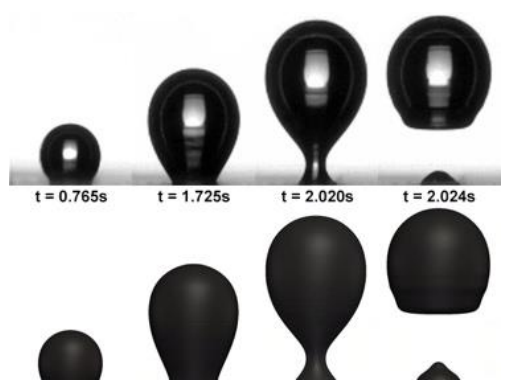

(a)

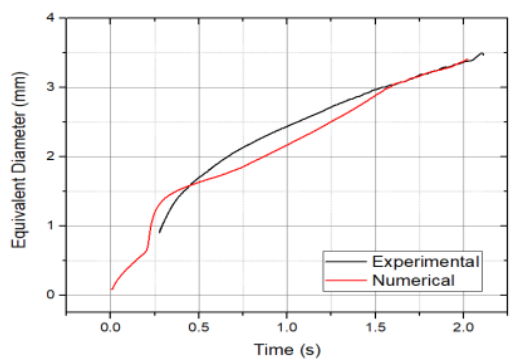

(b)

Fig. 1 Comparison between Experimental and Numerical results to validate the model. (a) Visual comparison of several bubble growth stages. (b) Equivalent diameter comparison. 
Having validated the model, a more in depth study on the velocity and temperature profiles inside the bubble can be made. The extracted colormaps for the studied case are shown in Figure 2. The temperature results portrait a hotter region near the surface and inside the bubble. On the bubble contact line, lower temperatures can be seen. Evaporation occurring in this area can justify this lower temperature values. Regarding the velocity results, the higher velocities can be seen during the detachment, in the neck region. The necking-detachment timeframe is very small so these should stand out as the bubble detaches.

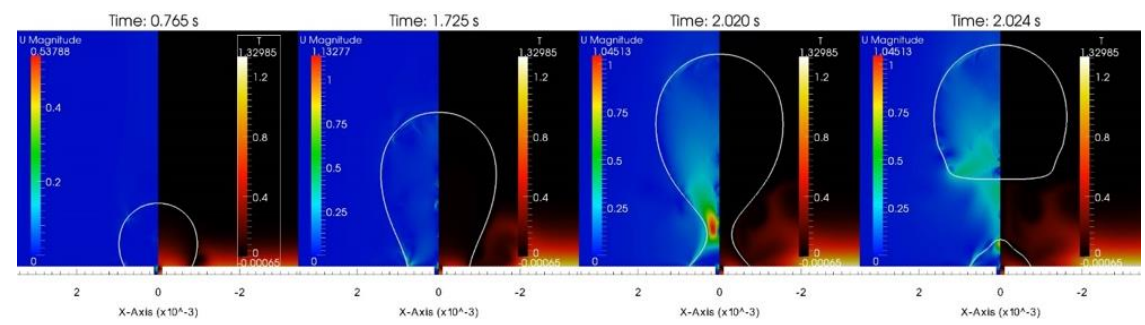

Fig 2. Velocity Magnitude and Temperature inside and outside the bubble.

\section{CONCLUSIONS}

The dynamic behavior of the bubble was accurately replicated by the model. Data on temperature and velocity profiles that cannot be extracted from the experimental measurements were post-processed from the simulation results. For a more complete validation of the model, further simulations need to be made testing different wettability as well as thermal conditions. In order also to investigate the thermal response of the surface, the conjugate heat transfer version of the utilised numerical simulation framework need to be applied in the future. In conclusion from the overall results of the present investigation it is obvious that the combination of enhanced direct numerical simulations with high-resolution transient experimental measurements can constitute a promising tool for further and deep understanding of the hydrodynamic and heat transfer characteristics of pool boiling in heated biphilic surfaces.

\section{ACKNOWLEDGEMENTS}

The authors from the IN+ team would like to acknowledge Fundação para a Ciência e Tecnologia for partially supporting the research under the framework of the project JICAM/0003/2017 and of project UTAP-EXPL/CTE/0064/2017. A. S. Moita also acknowledges FCT for financing her contract through the IF 2015 recruitment program (IF 00810-2015) and through the exploratory project associated with this contract. Dr. Anastasios Georgoulas would like to acknowledge the financial support from the Engineering and Physical Science Research Council in UK, through the grant EP/P013112/1.

\section{REFERENCES}

[1] Malavasi, I., Teodori, E., Moita, A.S., Moreira, A.L.N. and Marengo, M. Wettability effect on pool boiling: a review. Encyclopaedia of Two-Phase Heat Transfer and Flow III: Macro And Micro Flow Boiling And Numerical Modeling Fundamentals (A 4-volume Set), Vol. 4, Edited by J. Thome, World Scientific Publishing Co Pte Ltd. ISBN: 978-981-322731-6 (2018).

[2] Betz, A. R., Jenkins, J., Kim, C. and Attinger, D. Boiling heat transfer on superhydrophilic, superhydrophobic, and superbiphilic surfaces. Int. J. Heat Mass Transf. 57(2) (2013), 733-741.

[3] Jo, H., Park, H. S. and Kim, M. H., Single bubble dynamics on hydrophobic-hydrophilic mixed surfaces. In. J. Heat Mass Transf. 93(2016), 554-565.

[4] Georgoulas, A., Andredaki, M., and Marengo, M. An enhanced VOF method coupled with heat transfer and phase change to characterise bubble detachment in saturated pool boiling. Energies, 10(3) (2017).

[5] Teodori, E., Pontes, P., Moita, A.S., Moreira, A.L.N. Thermographic analysis of interfacial heat transfer mechanisms on droplet/wall interactions with high temporal and spatial resolution, Experimental Thermal and Fluid Science, 96(2018), 284294. 